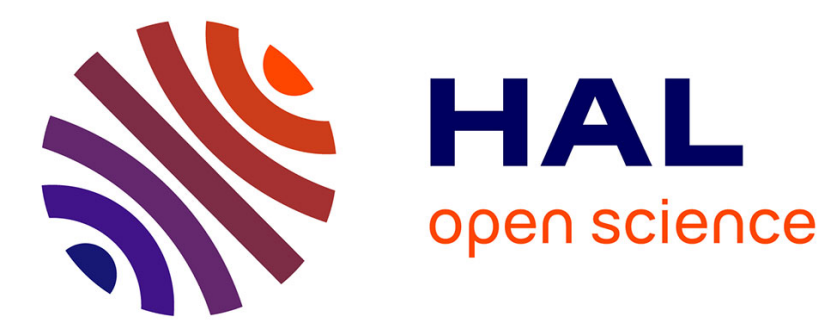

\title{
Tangential derivative of singular boundary integrals with respect to the position of collocation points.
}

\author{
Marc Bonnet, M. Guiggiani
}

\section{To cite this version:}

Marc Bonnet, M. Guiggiani. Tangential derivative of singular boundary integrals with respect to the position of collocation points.. Int. J. Num. Meth. in Eng., 1998, 41, pp.1255-1275. 10.1002/(SICI)1097-0207(19980415)41:73.0.CO;2-N . hal-00111250

\section{HAL Id: hal-00111250 \\ https://hal.science/hal-00111250}

Submitted on 11 Aug 2008

HAL is a multi-disciplinary open access archive for the deposit and dissemination of scientific research documents, whether they are published or not. The documents may come from teaching and research institutions in France or abroad, or from public or private research centers.
L'archive ouverte pluridisciplinaire HAL, est destinée au dépôt et à la diffusion de documents scientifiques de niveau recherche, publiés ou non, émanant des établissements d'enseignement et de recherche français ou étrangers, des laboratoires publics ou privés. 


\title{
Tangential derivative of singular boundary integrals with respect to the position of collocation points ${ }^{1}$
}

\author{
Marc BONNET \\ Laboratoire de Mécanique des Solides (URA CNRS 317) \\ Ecole Polytechnique, \\ 91128 Palaiseau cedex, France \\ email: bonnet@athena.polytechnique.fr \\ Massimo GUIGGIANI² \\ Dipartimento di Matematica, \\ Università degli Studi di Siena, \\ Via del Capitano 15, 53100 Siena, Italy \\ email: guiggiani@unisi.it
}

key words: sensitivity analysis, singular boundary integral equations,

hypersingular integrals, boundary element method

Revised version (with numerical examples), June 1997

${ }^{1}$ Int. J. Num. Meth. Engng. 41, 1255-1275 (1998)

${ }^{2}$ During the review process all correspondence should be addressed to Prof. M. Guiggiani, Dip. di Costruzioni Meccaniche e Nucl., via Diotisalvi 2, 56126 Pisa, Italy; ph. +39-50-585218, fax +39-50-585265 


\section{Abstract}

This paper investigates the evaluation of the sensitivity, with respect to tangential perturbations of the singular point, of boundary integrals having either weak or strong singularity. Both scalar potential and elastic problems are considered. A proper definition of the derivative of a strongly singular integral with respect to singular point perturbations should accommodate the concomitant perturbation of the vanishing exclusion neighbourhood involved in the limiting process used in the definition of the integral itself. This is done here by resorting to a shape sensitivity approach, considering a particular class of infinitesimal domain perturbations that "move" individual points, and especially the singular point, but leave the initial domain globally unchanged. This somewhat indirect strategy provides a proper mathematical setting for the analysis. Moreover, the resulting sensitivity expressions apply to arbitrary potential-type integrals with densities only subjected to some regularity requirements at the singular point, and thus are applicable to approximate as well as exact BEM solutions. Quite remarkable is the fact that the analysis is applicable when the singular point is located on an edge and simply continuous elements are used. The hypersingular BIE residual function is found to be equal to the derivative of the strongly singular BIE residual when the same values of the boundary variables are substituted in both SBIE and HBIE formulations, with interesting consequences for some error indicator computation strategies.

\section{Introduction}

Boundary integral equation (BIE) formulations lead to numerical techniques, namely boundary element methods (BEMs), that are now well established and widely applied. After a boundary value problem has been numerically solved using a standard boundary element method, it is sometimes necessary, or merely worthwhile, to be able to formulate and compute directional derivatives of field variables, or more generally of BIE residuals, at an observation point on the boundary and along a tangential direction. This of course leads to investigation of such derivatives for singular integrals of scalar or elastic potential theory. A relevant practical issue is the evaluation of in-plane strain, hoop stresses, etc. from a given (usually approximate) BEM solution. Another one concerns the evaluation of derivatives of BIE residuals under perturbations of the collocation points; a related idea is used to define local a posteriori error indicators in [8].

Concerning derivatives of the most singular integral operators encountered in standard BIE formulations (i.e. of the form of double-layer potentials), the Lyapunov-Tauber theorems of classical 
potential theory give results for the limit on the boundary of the normal derivative of scalar doublelayer potentials or of the traction operator applied to vector elastic potentials, see e.g. the books by Kellogg [11] and Kupradze et al. [12]. These results allow one to formulate Neumann problems using either single- or double-layer potentials. On the other hand, general results for tangent derivatives of strongly singular potential integrals are not given in the classical literature on potential theory.

More recently, many studies have been devoted to so-called hypersingular boundary integral equation (HBIE) formulations, which basically result from differentiation of strongly singular (SBIE) formulations with respect to components of the collocation point. Of course, both SBIE and HBIE give an identically zero residual (considered as a function of the collocation point) when the exact solution of a given problem is substituted into them. In practice, BIEs are discretized and collocated at a finite number of boundary points, and an approximate solution is obtained. For instance, the discrete SBIE is solved, and the SBIE residual may take nonzero values at boundary points that were not used for collocation. Then, if the previous approximate solution is substituted into the HBIE, is the HBIE residual function equal to the derivative of the SBIE function? This question is related to the study of e.g. local error estimation [8]. Its answer is not a priori obvious because both BIE formulations are defined in terms of a limiting process involving a vanishing exclusion neighbourhood of the singular point $\mathbf{y}$, so that in a proper definition of the derivative of a strongly singular integral with respect to y the vanishing neighbourhood should "move" with $\mathbf{y}$.

We address this particular difficulty in a somewhat indirect way, by resorting to a domain sensitivity approach. Specifically, the main idea used in this paper is the introduction of domain perturbations, depending on a time-like parameter $p$, that "move" individual points but leave the initial domain $(p=0)$ globally unchanged. This way, the concomitant change of singular point and exclusion neighbourhood is properly taken into account. The analysis then uses adequately chosen domain differentiation formulas. The sensitivity with respect to tangential perturbations of the singular point of boundary integrals having either weak or strong singularity, of the type used for both scalar and elastic BIE formulations, is formulated. From the results obtained, it is apparent that the HBIE residual function is indeed equal to the derivative of the SBIE residual function when the same values of the boundary variables are substituted in both SBIE and HBIE formulations. It is worth pointing out that this relationship between residuals issue is not raised in the formulation of hypersingular representations of potential gradients or stress tensors on the boundary, like in [6], although they allow one to evaluate tangential derivatives of the potential or displacement field. In particular the latter approach uses the fact that the density variables solve the relevant governing equations, whereas 
the present analysis addresses arbitrary potential-type integrals with densities only subjected to some (fairly weak) regularity requirements at the singular point. An advantage of this viewpoint is that the resulting formulas apply to approximate as well as exact BEM solutions, which is convenient in devising strategies for error indicator computation.

\section{Basic definitions and relations}

In this section we summarize some basic results that will be used extensively throughout the paper. More details can be found in previous papers dealing with shape sensitivity of BIE formulations [2], [3], [14] and in Appendix A of the recent book [1].

Let us consider, in a three-dimensional Euclidean space equipped with a Cartesian orthonormal basis $\left(\mathbf{e}_{1}, \mathbf{e}_{2}, \mathbf{e}_{3}\right)$, a body $\Omega_{p}$ whose shape depends on a time-like parameter $p$. The body $\Omega=\Omega_{0}$

corresponding to $p=0$ is traditionally assumed as the "initial" configuration. Points of $\Omega_{p}$ are denoted by $\mathbf{x}^{p}$ and $\mathbf{y}^{p}$, whereas points of $\Omega$ are simply denoted by $\mathbf{x}$ and $\mathbf{y}$.

Let the initial domain $\Omega$ be bounded by a Kellogg's regular surface $\partial \Omega=\Gamma$ with outer unit normal n [11]. On the boundary we can define some tangential differential operators. First, we define the tangential gradient $\nabla_{S} f$ of a scalar field $f$ defined on $\Omega$

$$
\nabla_{S} f(\mathbf{x}):=\nabla f-\mathbf{n}(\nabla f \cdot \mathbf{n})=\nabla f-\mathbf{n} \frac{\partial f}{\partial n}
$$

which is the projection of the gradient $\nabla f$ onto the tangent plane at $\mathbf{x} \in \partial \Omega$.

The $r$-th Cartesian component of $\nabla_{S} f$ can be denoted by $D_{r} f$ if we adopt the following definition for the tangential partial derivative $D_{r}$

$$
D_{r} f:=f_{, r}-n_{r} \frac{\partial f}{\partial n}=f_{, r}-n_{r}\left(f_{,_{m}} n_{m}\right),
$$

where the notation $f_{,_{m}}=\partial f / \partial x_{m}$ has been introduced.

The surface divergence $\operatorname{div}_{S} \mathbf{v}$ of a vector field $\mathbf{v}$ can now be defined as

$$
\operatorname{div}_{S} \mathbf{v}:=\nabla_{S} \cdot \mathbf{v}=D_{m} v_{m}
$$

It is convenient to define another tangential differential operator $D_{r s}$

$$
D_{r s} f:=n_{r} D_{s} f-n_{s} D_{r} f=n_{r} f,_{s}-n_{s} f,_{r} .
$$

Among the many versions of Stokes' theorem, we are mainly interested in the following ones

$$
\begin{aligned}
\int_{S} D_{r s} f d S & =e_{j r s} \oint_{\partial S} f \tau_{j} d s \\
\int_{S} D_{r} g d S & =\oint_{\partial S} g \nu_{r} d s-\int_{S} g n_{r} 2 \kappa d S
\end{aligned}
$$


where $\partial S$ is the contour of the (possibly) open surface $S$ and

$$
\kappa(\mathbf{x})=-\frac{1}{2} \operatorname{div}_{S} \mathbf{n}(\mathbf{x})=-\frac{1}{2} D_{a} n_{a}(\mathbf{x})
$$

is the mean curvature of $S$ at $\mathbf{x}$. The vector $\boldsymbol{\tau}$ is defined by

$$
\boldsymbol{\tau}=\mathbf{n} \times \boldsymbol{\nu},
$$

where $\boldsymbol{\nu}$ is the unit vector normal to $\partial S$ and tangent to $S$ and pointing towards the outside of $S . e_{j r s}$ denote the permutation symbols $\left(e_{123}=e_{231}=e_{312}=1, \quad e_{132}=e_{213}=e_{321}=-1\right.$, and equal to zero otherwise). Formula (6) is obtained by putting $f=g n_{s}$ in (5).

A geometrical transformation from $\Omega$ onto $\Omega_{p}$ is given by a mapping

$$
\mathbf{x} \in \Omega \rightarrow \mathbf{x}^{p}=\mathbf{\Phi}(\mathbf{x} ; p) \in \Omega_{p}
$$

where $\forall \mathbf{x} \in \Omega, \mathbf{\Phi}(\mathbf{x} ; 0)=\mathbf{x}$, as already stated. Similarly to continuum mechanics, it is assumed that any such mapping is a diffeomorphism between $\Omega$ and $\Omega_{p}$.

The corresponding initial transformation velocity $\boldsymbol{\theta}(\mathbf{x})$ is a vector field on $\Omega$ defined by

$$
\boldsymbol{\theta}(\mathbf{x}):=\left.\frac{\partial \Phi}{\partial p}\right|_{p=0}
$$

In other words, the geometrical transformation (8) can be expanded in the following form

$$
\mathbf{x}^{p}=\mathbf{x}+\boldsymbol{\theta}(\mathbf{x}) p+\mathbf{o}(p) .
$$

Now, let us consider a generic scalar field $f\left(p, \mathbf{x}^{p}\right)$ defined on the changing domain $\Omega_{p}$ and also depending explicitly on $p$. Its total derivative $f^{*}$ with respect to $p$, evaluated at $p=0$, is given by the following well-known expression

$$
\begin{aligned}
& \stackrel{*}{f}(\mathbf{x}):=\left.\frac{d f}{d p}\right|_{p=0}=\left.\left(\frac{\partial f}{\partial p}+f_{, m} \theta_{m}(\mathbf{x})\right)\right|_{p=0} \\
& =\left.(\dot{f}+\nabla f \cdot \boldsymbol{\theta}(\mathbf{x}))\right|_{p=0},
\end{aligned}
$$

where $\dot{f}=\partial f / \partial p$. Depending on the context, the total derivative $f^{*}$ can also be termed material or Lagrangian derivative. The function $\dot{f}$ can be consistently called Eulerian derivative.

The kernel functions that appear in boundary integral equations are two-point functions like $K\left(\mathbf{x}^{p}-\right.$ $\left.\mathbf{y}^{p}\right)$ which do not depend explicitly on $p$. Adopting the notation just defined, and assuming that both 


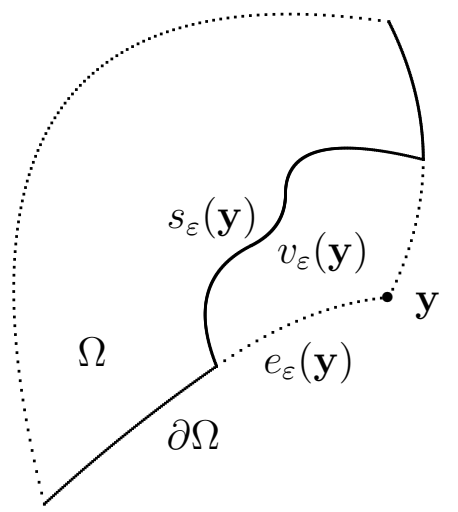

Figure 1: Exclusion of the singular point $\mathbf{y}$ by a vanishing neighbourhood $v_{\varepsilon}(\mathbf{y})$.

points follow the geometrical transformation (8), we have from (10) the following expression for the total derivative of $K$ with respect to $p$

$$
\stackrel{*}{K}(\mathbf{y}, \mathbf{x}):=\left.\frac{d K}{d p}\right|_{p=0}=K,_{m}(\mathbf{x}-\mathbf{y})\left[\theta_{m}(\mathbf{x})-\theta_{m}(\mathbf{y})\right],
$$

where $K_{m}=\partial K / \partial x_{m}=-\partial K / \partial y_{m}$.

The total derivative of the integral of a nonsingular function $f\left(p, \mathbf{x}^{p}\right)$ over a changing (possibly open) surface $S_{p}$ is given, from e.g. [13], by either of the formulas

$$
\begin{aligned}
\left.\frac{d}{d p}\left(\int_{S_{p}} f\left(p, \mathbf{x}^{p}\right) d S\right)\right|_{p=0} & =\int_{S}\left[* f+f \operatorname{div}_{S} \boldsymbol{\theta}\right] d S \\
& =\int_{S}\left[\dot{f}+\left(\frac{\partial f}{\partial n}-2 \kappa f\right)(\boldsymbol{\theta} \cdot \mathbf{n})\right] d S+\oint_{\partial S}(\boldsymbol{\theta} \cdot \boldsymbol{\nu}) f d s,
\end{aligned}
$$

where $S=S_{0}$. Formulas (12) and (13)) are equivalent; they relate the derivative of the integral to Lagrangian or Eulerian derivatives of the function $f$, respectively.

According to Petryk and Mroz [13], we can express the total (material) derivative of each Cartesian component of the unit normal $\mathbf{n}\left(\mathbf{x}^{p}\right)$ as

$$
\stackrel{*}{n}_{i}(\mathbf{x}):=\left.\frac{d n_{i}}{d p}\right|_{p=0}=-n_{m}\left(D_{i} \theta_{m}\right) .
$$

\section{$3 \quad$ Singular boundary integrals}

Singular boundary integrals arise naturally in the boundary element method (BEM) whenever the source (collocation) point $\mathbf{y}$ is taken to the boundary. Due to the singularity of the kernel functions $K$ involved, a limiting process is necessary for the definition of these integrals.

Let $\mathbf{y}$ be a fixed point on the boundary $\partial \Omega$ of a three-dimensional domain $\Omega$. We consider an exclusion neighbourhood $v_{\varepsilon}(\mathbf{y})$ of $\mathbf{y}$, of radius $\leq \varepsilon$ (Figure 1$)$. For any $\varepsilon>0, \mathbf{y}$ is always an external 
point for the domain $\Omega_{\varepsilon}(\mathbf{y})=\Omega-v_{\varepsilon}(\mathbf{y})$ whose boundary $\partial \Omega_{\varepsilon}$ is given by

$$
\partial \Omega_{\varepsilon}=\left(\partial \Omega-e_{\varepsilon}\right)+s_{\varepsilon}=\Gamma_{\varepsilon}+s_{\varepsilon}
$$

where $s_{\varepsilon}=\Omega \cap \partial v_{\varepsilon}, e_{\varepsilon}=\partial \Omega \cap \bar{v}_{\varepsilon}$, and $\Gamma_{\varepsilon}=\partial \Omega-e_{\varepsilon}$.

The typical singular boundary integral can be defined by means of a limiting process

$$
\mathcal{I}(\mathbf{y})=\lim _{\varepsilon \rightarrow 0} \int_{\Gamma_{\varepsilon}(\mathbf{y})+s_{\varepsilon}(\mathbf{y})} K(\mathbf{x}-\mathbf{y}) v(\mathbf{x}) d S_{x},
$$

where the density $v(\mathbf{x})$ is a sufficiently regular function.

There are instances (e.g., [8]) in which we may assume that the position of the source point $\mathbf{y}$ on $\partial \Omega$ depends on a time-like parameter $p$. Since the vanishing neighbourhood $v_{\varepsilon}(\mathbf{y})$ is attached to $\mathbf{y}$, the parameter $p$ also affects the surface of integration in (15) (although $p$ does not affect $\partial \Omega$ ). Somewhat similar considerations occur for the correct evaluation of the Cartesian derivatives of the integral representation for displacement at interior points $\mathbf{y}$ in the presence of initial strain [4]. Obviously, a necessary condition for the function $\mathbf{y}^{p}=\mathbf{y}(p)$ to be a diffeomorphism is that the point $\mathbf{y}$ lies either on a smooth part of $\partial \Omega$ or, at most, on an edge (two tangent planes). In the last case, the point $\mathbf{y}^{p}$ has to move along the edge itself.

Still following ref. [8] and for greater generality, the density function $v$ can be taken to explicitly depend on $p$ (this is the case, e.g., of approximate numerical solutions that clearly depend on the specific set of collocation points).

Hence, we can define the function $I(p)$

$$
I(p)=\lim _{\varepsilon \rightarrow 0} \int_{\Gamma_{\varepsilon}\left(\mathbf{y}^{p}\right)+s_{\varepsilon}\left(\mathbf{y}^{p}\right)} K\left(\mathbf{x}-\mathbf{y}^{p}\right) v(p, \mathbf{x}) d S_{x},
$$

which can be seen as $\mathcal{I}\left(\mathbf{y}^{p}\right)$ with a $p$-dependent density function.

In this paper we address the problem of the evaluation of the derivative

$$
\left.\frac{d I}{d p}\right|_{p=0} .
$$

Derivatives of this type are basically what are required for the evaluation of the sensitivity of an approximate BEM solution with respect to the position of the collocation points [8].

\section{Tangential derivatives of singular boundary integrals}

To evaluate the derivative $d I / d p$ we rely on a domain sensitivity approach. Although in the present context neither the shape of the body nor the discretization are affected by the parameter $p$, the 
sensitivity viewpoint provides a convenient mathematical means to describe exclusion neighbourhoods $v_{\varepsilon}(\mathbf{y})$ moving according to perturbations of the singular point $\mathbf{y}$.

To this end, let us consider a geometrical transformation $\mathbf{x} \rightarrow \mathbf{\Phi}(\mathbf{x}, p)$ as in (8) but with initial transformation velocity $\boldsymbol{\theta}$ (defined in (9)) such that

$$
\boldsymbol{\theta}(\mathbf{x}) \cdot \mathbf{n}(\mathbf{x})=0 \quad(\forall \mathbf{x} \in \partial \Omega), \quad \text { and } \quad \boldsymbol{\theta}(\mathbf{y}) \neq 0
$$

In other words, the transformation velocity is almost everywhere orthogonal to the boundary normal of the body. Under any such transformation, $\Omega$ is globally invariant (up to order $O(p)$ ), while

$$
\mathbf{y} \rightarrow \mathbf{y}+\mathbf{a} p+\mathbf{o}(p)
$$

where

$$
\mathbf{a}=\boldsymbol{\theta}(\mathbf{y})
$$

is the transformation velocity of the source point. Therefore, the initial (i.e. of first-order with respect to $p$ about $p=0$ ) effect of the perturbation of the position of the singular point $\mathbf{y}$ on $I(p)$ is adequately taken into account. At the same time, $v_{\varepsilon}(\mathbf{y})$ is "moved" (and possibly distorted, but this is irrelevant) along with the singular point, so that the singular integral (16) is properly defined for small $p \geq 0$.

Notice that if $\partial \Omega$ has more than two tangent planes at $\mathbf{y}$, then there are no transformation velocity fields such that $\mathbf{a} \cdot \mathbf{n}(\mathbf{y})=0$.

Essentially, with a transformation satisfying (18) we can use all the results already available from sensitivity analysis, yet we are only considering the effect of the perturbation of the singular point $\mathbf{y}$ and of the attached neighbourhood $v_{\varepsilon}$. Formulas for the derivative of an integral over a changing surface were given in (12) and (13). The derivative of (16) can be conveniently evaluated applying formula (12) to the integral on $s_{\varepsilon}$ and formula (13) to the integral on $\Gamma_{\varepsilon}$ (all derivatives are understood at $p=0)$

$$
\frac{d I}{d p}=\lim _{\varepsilon \rightarrow 0}\left\{\int_{s_{\varepsilon}}\left[(K v)^{*}+K v D_{m} \theta_{m}\right] d S+\int_{\Gamma_{\varepsilon}}(K v)^{\bullet} d S+\oint_{\partial \Gamma_{\varepsilon}}\left(\theta_{m} \nu_{m}\right) K v d s\right\} .
$$

In this way, full advantage is taken from the orthogonality condition (18).

Although quite obvious, we may also observe that

$$
(K v)^{*}=\stackrel{*}{K} v+K^{*} v=K_{m}\left[\theta_{m}(\mathbf{x})-a_{m}\right] v+K^{*},
$$

and

$$
(K v)^{\cdot}=\dot{K} v+K \dot{v}=\frac{\partial K}{\partial y_{m}} a_{m} v+K \dot{v}=-K,_{m} a_{m} v+K \dot{v}
$$


In this paper we consider the tangential derivative of singular integrals with respect to the source point $\mathbf{y}$ for three-dimensional potential and elastic problems. It is worth recalling that the analysis is not restricted to smooth boundaries, but also covers the case of $\mathbf{y}$ on an edge. In Appendix $\mathrm{A}$ it is shown that the final results are not affected by the shape of $v_{\varepsilon}$. Therefore, it is always assumed to employ a symmetric neighbourhood $v_{\varepsilon}(\mathbf{y})$ of uniform radius $\varepsilon$.

\section{$5 \quad$ Weakly singular kernels}

The formulation of cartesian derivatives of single-layer scalar or elastic potentials has been studied in classical potential theory. The results of this section are in effect nothing more than extensions to $p$-dependent density functions of classical results on cartesian derivatives of single-layer scalar or elastic potentials, see e.g. [11] for scalar potentials and [12] for elasticity. However, it is interesting to see the present sensitivity approach at work in this context before dealing with the more difficult case of double-layer potentials.

\subsection{Potential problems}

Let us consider the following boundary integral with weakly singular kernel

$$
I(p)=\lim _{\varepsilon \rightarrow 0} \int_{\Gamma_{\varepsilon}\left(\mathbf{y}^{p}\right)+s_{\varepsilon}\left(\mathbf{y}^{p}\right)} G\left(\mathbf{x}-\mathbf{y}^{p}\right) q(p, \mathbf{x}) d S_{x}
$$

where the kernel $K=G=1 /(4 \pi r)$, with $r=|\mathbf{x}-\mathbf{y}|$, is the fundamental solution for 3D potential problems, and the density function $q$ is a sufficiently regular function (usually an approximation to the normal derivative of the potential).

A first expression for $d I / d p$ is given by $(20)$, where $(G q)^{\bullet}$ can be expanded according to (22)

$$
\frac{d I}{d p}=\lim _{\varepsilon \rightarrow 0}\left\{\int_{s_{\varepsilon}}\left[(G q)^{*}+G q D_{m} \theta_{m}\right] d S+\int_{\Gamma_{\varepsilon}}\left[G \dot{q}+a_{m} \frac{\partial G}{\partial y_{m}} q\right] d S+\oint_{\partial \Gamma_{\varepsilon}}\left(\theta_{m} \nu_{m}\right) G q d s\right\} .
$$

For $3 \mathrm{D}$ problems we have on $s_{\varepsilon}$ that $G=O\left(\varepsilon^{-1}\right), G_{m}=O\left(\varepsilon^{-2}\right)$, and $\theta_{m}(\mathbf{x})-a_{m}=O\left(\varepsilon^{\alpha}\right)$, with $\alpha>0$, because of the regularity assumption on the geometrical transformation $\boldsymbol{\Phi}$. If we also assume that $|*(\mathbf{x})-\stackrel{*}{q}(\mathbf{y})| \leq C r^{\alpha-1}$ (which is quite reasonable), we can conclude that

$$
\lim _{\varepsilon \rightarrow 0} \int_{s_{\varepsilon}}\left[(G q)^{*}+G q D_{m} \theta_{m}\right] d S=0 .
$$

Moreover, when $\mathbf{y}$ is on an edge of tangent $\mathbf{a}$, the limit of the line integral on $\partial \Gamma_{\varepsilon}$ is zero

$$
\lim _{\varepsilon \rightarrow 0}\left[\oint_{\partial \Gamma_{\varepsilon}}\left(\theta_{m}-a_{m}\right) \nu_{m} G q d s+a_{m} \oint_{\partial \Gamma_{\varepsilon}} \nu_{m} G q d s\right]=0
$$


since it is zero on each side (provided $q$ is piecewise $C^{0, \alpha}$ at $\mathbf{y}$ ). Essentially, owing to the scalar product $a_{m} \nu_{m}$, the line integral on each side amounts in the limit to the integral from 0 to $\pi$ of $\cos \varphi d \varphi$.

Therefore, we have that

$$
\begin{aligned}
\left.\frac{d I}{d p}\right|_{p=0} & =\lim _{\varepsilon \rightarrow 0}\left\{\int_{\Gamma_{\varepsilon}}\left[G \dot{q}+a_{m} \frac{\partial G}{\partial y_{m}} q\right] d S\right\} \\
& =\int_{\partial \Omega} G \dot{q} d S+a_{m} f_{\partial \Omega} W_{m} q d S \\
& =\int_{\partial \Omega} G \dot{q} d S+f_{\partial \Omega} \dot{G} q d S
\end{aligned}
$$

where $W_{m}=\partial G / \partial y_{m}$ are the kernel functions of the hypersingular boundary integral equations [10] and $\dot{G}=a_{m} W_{m}$. The weak singularity of $G$ allows the first integral in (25) to be defined as an ordinary integral on $\partial \Omega$. On the other hand, the second integral with strongly singular kernel $\dot{G}$ is equivalent (owing to the assumed circular shape of $\partial \Gamma_{\varepsilon}$ ) to a Cauchy principal value [5].

\subsection{Elastic problems}

In this case we have to consider the tangential derivative of the weakly singular integral

$$
I(p)=\lim _{\varepsilon \rightarrow 0} \int_{\Gamma_{\varepsilon}\left(\mathbf{y}^{p}\right)+s_{\varepsilon}\left(\mathbf{y}^{p}\right)} U_{i j}\left(\mathbf{x}-\mathbf{y}^{p}\right) t_{j}(p, \mathbf{x}) d S_{x},
$$

where the kernels $U_{i j}$, are the fundamental solutions for 3D elastic problems, and the density functions $t_{j}$ are just sufficiently regular functions. Again, a possible expression for $d I / d p$ is given by (20).

Using the same arguments as above, we can show that

$$
\lim _{\varepsilon \rightarrow 0} \int_{s_{\varepsilon}}\left[\left(U_{i j} t_{j}\right)^{*}+U_{i j} t_{j} D_{m} \theta_{m}\right] d S=0
$$

Similarly, when $\mathbf{y}$ is on an edge of tangent $\mathbf{a}$ the line integral on $\partial \Gamma_{\varepsilon}$ is zero since it is zero on each side (provided, on each element, $t_{j} \in C^{0, \alpha}$ at $\mathbf{y}$ ).

Therefore, we have that

$$
\begin{aligned}
\left.\frac{d I}{d p}\right|_{p=0} & =\lim _{\varepsilon \rightarrow 0}\left\{\int_{\Gamma_{\varepsilon}}\left[U_{i j} \dot{t}_{j}+a_{m} \frac{\partial U_{i j}}{\partial y_{m}} t_{j}\right] d S\right\} \\
& =\int_{\partial \Omega} U_{i j} \dot{t}_{j} d S+a_{m} f_{\partial \Omega} W_{i j m} t_{j} d S \\
& =\int_{\partial \Omega} U_{i j} \dot{t}_{j} d S+f_{\partial \Omega} \dot{U}_{i j} t_{j} d S
\end{aligned}
$$

where $W_{i j m}=\partial U_{i j} / \partial y_{m}$ and $\dot{U}_{i j}=a_{m} W_{i j m}$. 
Formulas (27) and (30) have the same structure. They show that the derivative $d I / d p$ is given by a term that accounts for the dependence on $p$ of the density function and another term that takes into account the effect of the changing singular point on the kernel function. This last term is the one predicted by classical potential theory.

\section{$6 \quad$ Strongly singular kernels}

\subsection{Potential problems}

Let us consider the following boundary integral with strongly singular kernel $T=G,{ }_{j} n_{j}$

$$
I(p)=\lim _{\varepsilon \rightarrow 0} \int_{\Gamma_{\varepsilon}\left(\mathbf{y}^{p}\right)+s_{\varepsilon}\left(\mathbf{y}^{p}\right)} T\left(\mathbf{y}^{p}, \mathbf{x}\right) u(p, \mathbf{x}) d S_{x}=\int_{\Gamma_{\varepsilon}\left(\mathbf{y}^{p}\right)+s_{\varepsilon}\left(\mathbf{y}^{p}\right)} G,{ }_{j}\left(\mathbf{x}-\mathbf{y}^{p}\right) n_{j}(\mathbf{x}) u(p, \mathbf{x}) d S_{x},
$$

where, in general, the density function $u$ is only an approximation to a potential.

First we consider the derivative of the integral on $s_{\varepsilon}$, for fixed $\varepsilon>0$. According to (20), we apply formula (12) and obtain

$$
\left.\frac{d}{d p}\left(\int_{s_{\varepsilon}\left(\mathbf{y}^{p}\right)} T u d S\right)\right|_{p=0}=\int_{s_{\varepsilon}(\mathbf{y})}\left[(T u)^{*}+T u D_{i} \theta_{i}\right] d S,
$$

This expression can be given a more explicit form using (11) and (14)

$$
\begin{aligned}
(T u)^{*}+T u D_{i} \theta_{i} & =\left(G,{ }_{j} n_{j} u\right)^{*}+G,{ }_{j} n_{j} u D_{i} \theta_{i} \\
& =G,{ }_{j} n_{j} \stackrel{*}{u}+\stackrel{*}{G},{ }_{j} n_{j} u+G,{ }_{j}{ }_{j} u+G,{ }_{j} n_{j} u D_{i} \theta_{i} \\
& =G,{ }_{j} n_{j} \stackrel{*}{u}+\left[G,{ }_{i j} n_{j}\left(\theta_{i}-a_{i}\right)+G,{ }_{j}\left(n_{j} D_{i} \theta_{i}-n_{i} D_{j} \theta_{i}\right)\right] u \\
& =G,{ }_{j} n_{j} \stackrel{*}{u}+\left[\left(D_{j i} G,{ }_{j}\right)\left(\theta_{i}-a_{i}\right)+G,{ }_{j} D_{j i} \theta_{i}\right] u \\
& =G,{ }_{j} n_{j} \stackrel{*}{u}+D_{j i}\left(G,{ }_{j}\left[\theta_{i}-a_{i}\right]\right) u
\end{aligned}
$$

where $a_{i}=\theta_{i}(\mathbf{y})$. Expression (36) comes from (4) once with $f=\theta_{i}$, and once with $f=G,{ }_{j}$ with the additional fact that $G,_{i i}=0$. The final step is possible since $D_{j i} a_{i}=0$ ( $a_{i}$ do not depend on $\left.\mathbf{x}\right)$.

We can now add and subtract the same terms to obtain

$$
\begin{gathered}
(T u)^{*}+T u D_{i} \theta_{i}=G,{ }_{j} n_{j}[\stackrel{*}{u}(\mathbf{x})-\stackrel{*}{u}(\mathbf{y})]+D_{j i}\left(G,{ }_{j}\left[\theta_{i}-a_{i}\right]\right)[u(\mathbf{x})-u(\mathbf{y})] \\
+\stackrel{*}{u}(\mathbf{y}) G,{ }_{j} n_{j}+u(\mathbf{y}) D_{j i}\left(G,{ }_{j}\left[\theta_{i}-a_{i}\right]\right) .
\end{gathered}
$$

At this stage we have to make some assumptions on the regularity of the density function $u(\mathbf{x})$ at y. We assume $u \in C^{0, \alpha}(\mathbf{y})$ and $\stackrel{*}{u} \in C^{0, \alpha}(\mathbf{y})$, along with $\boldsymbol{\theta} \in C^{0,1}(\mathbf{y})$ which comes from the geometrical 
transformation $\boldsymbol{\Phi}$ being a diffeomorphism. It is worth noting that we do not require $u \in C^{1, \alpha}(\mathbf{y})$ (i.e., $\left.\nabla u \in C^{0, \alpha}(\mathbf{y})\right)$, but only the total derivative

$$
\stackrel{*}{u}(\mathbf{y})=\dot{u}(\mathbf{y})+\nabla u \cdot \mathbf{a}
$$

to be Hölder continuous at $\mathbf{y}$. This is a condition that is satisfied by standard continuous boundary elements provided $\mathbf{a}$ is tangent to the interelement border between two elements.

Under these assumptions, the integrals on $s_{\varepsilon}$ of the first two terms on the r.h.s. of eq. (38) vanish in the limit, as $\varepsilon \rightarrow 0$.

Therefore, we have that

$$
\int_{s_{\varepsilon}(\mathbf{y})}\left[(T u)^{*}+T u D_{i} \theta_{i}\right] d S=\stackrel{*}{u}(\mathbf{y}) \int_{s_{\varepsilon}(\mathbf{y})} G,{ }_{j} n_{j} d S+u(\mathbf{y}) \int_{s_{\varepsilon}(\mathbf{y})} D_{j i}\left(G,,_{j}\left[\theta_{i}-a_{i}\right]\right) d S+o(1) .
$$

The limit of the first integral in the above expression is readily obtained

$$
\lim _{\varepsilon \rightarrow 0} \stackrel{*}{u}(\mathbf{y}) \int_{s_{\varepsilon}(\mathbf{y})} G,{ }_{j} n_{j} d S=\stackrel{*}{u}(\mathbf{y}) \lim _{\varepsilon \rightarrow 0} \int_{s_{\varepsilon}(\mathbf{y})} T d S=\stackrel{*}{u}(\mathbf{y}) c(\mathbf{y})=\left[\dot{u}(\mathbf{y})+\frac{\partial u}{\partial a}\right] c(\mathbf{y}),
$$

where, by definition, $c(\mathbf{y})$ is the free-term coefficient of the usual boundary integral equation.

We already found that $D_{j i} G,,_{j}=G,{ }_{i j} n_{j}$. Hence from the final term in (40) we get

$$
\begin{aligned}
-u(\mathbf{y}) a_{i} \int_{s_{\varepsilon}(\mathbf{y})} D_{j i} G,{ }_{j} d S & =-u(\mathbf{y}) a_{i} \int_{s_{\varepsilon}(\mathbf{y})} G,{ }_{i j} n_{j} d S \\
& =u(\mathbf{y}) a_{i} \int_{s_{\varepsilon}(\mathbf{y})} V_{i} d S \\
& =u(\mathbf{y}) a_{i}\left[\frac{b_{i}}{\varepsilon}+d_{i}+O(\varepsilon)\right],
\end{aligned}
$$

that is the same free-term coefficients of the hypersingular boundary integral equations as obtained in [7]. In fact the hypersingular kernel is $V_{i}=-G,{ }_{i j} n_{j}$.

Stokes' theorem in the form (5) can be applied to the function $D_{j i}\left(G,{ }_{j} \theta_{i}\right)$ in (40)

$$
u(\mathbf{y}) \int_{s_{\varepsilon}(\mathbf{y})} D_{j i}\left(G,{ }_{j} \theta_{i}\right) d S=u(\mathbf{y}) e_{j i p} \oint_{\partial s_{\varepsilon}} G,{ }_{j} \theta_{i} \tilde{\tau}_{p} d s=-u(\mathbf{y}) \oint_{\partial \Gamma_{\varepsilon}} T \theta_{i} \nu_{i} d s
$$

where we used the fact that $\partial s_{\varepsilon}$ and $\partial \Gamma_{\varepsilon}$ are identical curves with opposite tangents $\tilde{\boldsymbol{\tau}}=-\boldsymbol{\tau}$. The last step in (43) comes from the following general result

$$
e_{j i p} w_{i} \tilde{\tau}_{p}=-(\mathbf{w} \times \boldsymbol{\tau})_{j}=-(\mathbf{w} \cdot \boldsymbol{\nu}) n_{j}+(\mathbf{w} \cdot \mathbf{n}) \nu_{j}
$$

where $\mathbf{w}$ is a general vector, $\tilde{\boldsymbol{\tau}}=-\boldsymbol{\tau}$, and $(\mathbf{n}, \boldsymbol{\nu}, \boldsymbol{\tau})$ is a Cartesian orthonormal basis defined in (7). If the vector $\mathbf{w}$ is replaced by $\boldsymbol{\theta}$ we have that

$$
e_{j i p} \theta_{i} \tilde{\tau}_{p}=-(\boldsymbol{\theta} \times \boldsymbol{\tau})_{j}=-(\boldsymbol{\theta} \cdot \boldsymbol{\nu}) n_{j}
$$


because of the assumed orthogonality between $\boldsymbol{\theta}$ and $\mathbf{n}$ (eq. (18)).

Collecting the results of (41), (42), and (43), we can write the derivative of the integral on $s_{\varepsilon}$ as

$$
\begin{aligned}
\left.\frac{d}{d p}\left(\int_{s_{\varepsilon}\left(\mathbf{y}^{p}\right)} T u d S\right)\right|_{p=0} & =\int_{s_{\varepsilon}(\mathbf{y})}\left[(T u)^{*}+T u D_{i} \theta_{i}\right] d S \\
& =\left[\dot{u}(\mathbf{y})+\frac{\partial u}{\partial a}\right] c(\mathbf{y})+u(\mathbf{y}) a_{i}\left[\frac{b_{i}}{\varepsilon}+d_{i}+O(\varepsilon)\right]-u(\mathbf{y}) \oint_{\partial \Gamma_{\varepsilon}} T \theta_{i} \nu_{i} d s .
\end{aligned}
$$

We can now turn our attention to the integral on $\Gamma_{\varepsilon}$ in (31). To compute the derivative with respect to $p$, for fixed $\varepsilon$, we can employ the general relation (13) with the obvious simplification due to $\boldsymbol{\theta} \cdot \mathbf{n}=0$ on $\Gamma_{\varepsilon}$

$$
\begin{aligned}
\left.\frac{d}{d p}\left(\int_{\Gamma_{\varepsilon}} T u d S\right)\right|_{p=0} & =\int_{\Gamma_{\varepsilon}} T \dot{u} d S+\int_{\Gamma_{\varepsilon}} \dot{T} u d S+\oint_{\partial \Gamma_{\varepsilon}} T u \theta_{i} \nu_{i} d s \\
& =\int_{\Gamma_{\varepsilon}} T \dot{u} d S-a_{i} \int_{\Gamma_{\varepsilon}}\left(G, j_{i} n_{j}\right) u d S+u(\mathbf{y}) \oint_{\partial \Gamma_{\varepsilon}} T \theta_{i} \nu_{i} d s+\oint_{\partial \Gamma_{\varepsilon}} T[u-u(\mathbf{y})] \theta_{i} \nu_{i} d s \\
& =\int_{\Gamma_{\varepsilon}} T \dot{u} d S+a_{i} \int_{\Gamma_{\varepsilon}} V_{i} u d S+u(\mathbf{y}) \oint_{\partial \Gamma_{\varepsilon}} T \theta_{i} \nu_{i} d s+o(1)
\end{aligned}
$$

where $\dot{T}=a_{i} V_{i}$.

When contributions from $s_{\varepsilon}$ and $\Gamma_{\varepsilon}$ are taken together, we observe that the line integrals in (44) and (45) cancel each other. Therefore, we are left with the following final expression

$$
\begin{aligned}
& \left.\frac{d I}{d p}\right|_{p=0}=\left.\lim _{\varepsilon \rightarrow 0} \frac{d}{d p}\left[\int_{\Gamma_{\varepsilon}\left(\mathbf{y}^{p}\right)+s_{\varepsilon}\left(\mathbf{y}^{p}\right)} T u d S\right]\right|_{p=0} \\
& =\lim _{\varepsilon \rightarrow 0}\left[*(\mathbf{y}) \int_{s_{\varepsilon}} T d S+\int_{\Gamma_{\varepsilon}} T \dot{u} d S+u(\mathbf{y}) \int_{s_{\varepsilon}} \dot{T} d S+\int_{\Gamma_{\varepsilon}} \dot{T} u d S+a_{i} \oint_{\partial \Gamma_{\varepsilon}} T[u-u(\mathbf{y})] \nu_{i} d s\right] \\
& =\lim _{\varepsilon \rightarrow 0}\left[\left(\dot{u}(\mathbf{y})+\frac{\partial u}{\partial a}\right) \int_{s_{\varepsilon}} T d S+\int_{\Gamma_{\varepsilon}} T \dot{u} d S+u(\mathbf{y}) a_{i} \int_{s_{\varepsilon}} V_{i} d S+a_{i} \int_{\Gamma_{\varepsilon}} V_{i} u d S\right] \\
& =\dot{u}(\mathbf{y}) c(\mathbf{y})+\frac{\partial u}{\partial a} c(\mathbf{y})+f_{\partial \Omega} T \dot{u} d S+\lim _{\varepsilon \rightarrow 0}\left[a_{i} \int_{\Gamma_{\varepsilon}} V_{i} u d S+u(\mathbf{y}) a_{i}\left(\frac{b_{i}}{\varepsilon}+d_{i}\right)\right] .
\end{aligned}
$$

We recall that the above expression has been obtained by assuming $u$ and only its directional derivative $\partial u / \partial a$ to be $C^{0, \alpha}$ at the point $\mathbf{y}$ lying on an edge of tangent $\mathbf{a}$. This is typically satisfied between two standard continuous boundary elements. 


\subsection{Elastic problems}

Let us consider the following boundary integral with strongly singular kernel $T_{i j}=\Sigma_{i j k} n_{k}$, where $\Sigma_{i j k}$ is the stress tensor associated to the displacement field $U_{i j}$ (fixed index $i$ )

$$
\begin{aligned}
I(p) & =\lim _{\varepsilon \rightarrow 0} \int_{\Gamma_{\varepsilon}\left(\mathbf{y}^{p}\right)+s_{\varepsilon}\left(\mathbf{y}^{p}\right)} T_{i j}\left(\mathbf{y}^{p}, \mathbf{x}\right) u_{j}(p, \mathbf{x}) d S_{x} \\
& =\lim _{\varepsilon \rightarrow 0} \int_{\Gamma_{\varepsilon}\left(\mathbf{y}^{p}\right)+s_{\varepsilon}\left(\mathbf{y}^{p}\right)} \Sigma_{i j k}\left(\mathbf{x}-\mathbf{y}^{p}\right) n_{k}(\mathbf{x}) u_{j}(p, \mathbf{x}) d S_{x}
\end{aligned}
$$

where, in general, the density functions $u_{j}$ are only an approximation to displacement field components.

As for the potential case, we consider first the derivative of the integral on $s_{\varepsilon}$, for fixed $\varepsilon>0$. According to (20), we apply formula (12) and obtain

$$
\left.\frac{d}{d p}\left(\int_{s_{\varepsilon}\left(\mathbf{y}^{p}\right)} T_{i j} u_{j} d S\right)\right|_{p=0}=\int_{s_{\varepsilon}(\mathbf{y})}\left[\left(T_{i j} u_{j}\right)^{*}+T_{i j} u_{j} D_{\ell} \theta_{\ell}\right] d S
$$

This expression can be given a more explicit form using (11) and (14)

$$
\begin{aligned}
\left(T_{i j} u_{j}\right)^{*}+T_{i j} u_{j} D_{\ell} \theta_{\ell} & =\left(\Sigma_{i j k} n_{k} u_{j}\right)^{*}+\Sigma_{i j k} n_{k} u_{j} D_{\ell} \theta_{\ell} \\
& =\Sigma_{i j k} n_{k} \stackrel{*}{u}_{j}+\stackrel{*}{\Sigma}_{i j k} n_{k} u_{j}+\Sigma_{i j k} \stackrel{*}{n}_{k} u_{j}+\Sigma_{i j k} n_{k} u_{j} D_{\ell} \theta_{\ell} \\
& =\Sigma_{i j k} n_{k} \stackrel{*}{u}_{j}+\left[\Sigma_{i j k, \ell} n_{k}\left(\theta_{\ell}-a_{\ell}\right)+\Sigma_{i j k}\left(n_{k} D_{\ell} \theta_{\ell}-n_{\ell} D_{k} \theta_{\ell}\right)\right] u_{j} \\
& =\Sigma_{i j k} n_{k} \stackrel{*}{u}_{j}+\left[\left(D_{k \ell} \Sigma_{i j k}\right)\left(\theta_{\ell}-a_{\ell}\right)+\Sigma_{i j k} D_{k \ell} \theta_{\ell}\right] u_{j} \\
& =\Sigma_{i j k} n_{k} \stackrel{*}{u}_{j}(\mathbf{x})+D_{k \ell}\left(\Sigma_{i j k}\left[\theta_{\ell}-a_{\ell}\right]\right) u_{j}(\mathbf{x})
\end{aligned}
$$

Expression (52) comes from (4) once with $f=\theta_{\ell}$, and once with $f=\Sigma_{i j k}$ with the additional fact that $\Sigma_{i j k, k}=0$.

Following the development of (38) in the potential case, we can now add and subtract the same terms in (53) which, along with the assumptions $u_{j} \in C^{0, \alpha}(\mathbf{y})$ and $\stackrel{*}{u}_{j} \in C^{0, \alpha}(\mathbf{y})$, allows us to write (cfr. eq. (40))

$$
\int_{s_{\varepsilon}(\mathbf{y})}\left[\left(T_{i j} u_{j}\right)^{*}+T_{i j} u_{j} D_{\ell} \theta_{\ell}\right] d S=\stackrel{*}{u}_{j}(\mathbf{y}) \int_{s_{\varepsilon}(\mathbf{y})} \Sigma_{i j k} n_{k} d S+u_{j}(\mathbf{y}) \int_{s_{\varepsilon}(\mathbf{y})} D_{k \ell}\left(\Sigma_{i j k}\left[\theta_{\ell}-a_{\ell}\right]\right) d S+o(1) .
$$

The limit of the first integral on the r.h.s. of the above expression is readily obtained

$$
\lim _{\varepsilon \rightarrow 0} \stackrel{*}{u}_{j}(\mathbf{y}) \int_{s_{\varepsilon}(\mathbf{y})} \Sigma_{i j k} n_{k} d S=\stackrel{*}{u}_{j}(\mathbf{y}) \lim _{\varepsilon \rightarrow 0} \int_{s_{\varepsilon}(\mathbf{y})} T_{i j} d S=\stackrel{*}{u}_{j}(\mathbf{y}) c_{i j}(\mathbf{y})=\left[\dot{u}_{j}(\mathbf{y})+\frac{\partial u_{j}}{\partial a}\right] c_{i j}(\mathbf{y}),
$$

where, by definition, $c_{i j}(\mathbf{y})$ are the free-term coefficients of the usual boundary integral equation. 
The last term in (54) can be converted in a more convenient form if we observe that $D_{k \ell} \Sigma_{i j k}=$ $\Sigma_{i j k, \ell} n_{k}$

$$
\begin{aligned}
-u_{j}(\mathbf{y}) a_{\ell} \int_{s_{\varepsilon}(\mathbf{y})} D_{k \ell} \Sigma_{i j k} d S & =-u_{j}(\mathbf{y}) a_{\ell} \int_{s_{\varepsilon}(\mathbf{y})} \Sigma_{i j k, \ell} n_{k} d S \\
& =u_{j}(\mathbf{y}) a_{\ell} \int_{s_{\varepsilon}(\mathbf{y})} V_{i j \ell} d S \\
& =u_{j}(\mathbf{y}) a_{\ell}\left[\frac{b_{i j \ell}}{\varepsilon}+d_{i j \ell}+O(\varepsilon)\right],
\end{aligned}
$$

that is the same free-term coefficients of the hypersingular boundary integral equations as obtained in [7]. In fact the hypersingular kernel is $V_{i j \ell}=-\Sigma_{i j k, \ell} n_{k}$.

Finally, Stokes' theorem in the form (5) can be applied to the function $D_{k \ell}\left(\Sigma_{i j k} \theta_{\ell}\right)$ in (54)

$$
u_{j}(\mathbf{y}) \int_{s_{\varepsilon}(\mathbf{y})} D_{k \ell}\left(\Sigma_{i j k} \theta_{\ell}\right) d S=u_{j}(\mathbf{y}) e_{k \ell p} \oint_{\partial s_{\varepsilon}} \Sigma_{i j k} \theta_{\ell} \tilde{\tau} d s=-u_{j}(\mathbf{y}) \oint_{\partial \Gamma_{\varepsilon}} T_{i j} \theta_{\ell} \nu_{\ell} d s
$$

where we took exactly the same steps as in (43).

Summing up, the derivative of the integral on $s_{\varepsilon}$ can be written as

$$
\begin{aligned}
& \left.\frac{d}{d p}\left(\int_{s_{\varepsilon}\left(\mathbf{y}^{p}\right)} T_{i j} u_{j} d S\right)\right|_{p=0}=\int_{s_{\varepsilon}(\mathbf{y})}\left[\left(T_{i j} u_{j}\right)^{*}+T_{i j} u_{j} D_{\ell} \theta_{\ell}\right] d S \\
& =\left[\dot{u}_{j}(\mathbf{y})+\frac{\partial u_{j}}{\partial a}\right] c_{i j}(\mathbf{y})+u_{j}(\mathbf{y}) a_{\ell}\left[\frac{b_{i j \ell}}{\varepsilon}+d_{i j \ell}+O(\varepsilon)\right]-u_{j}(\mathbf{y}) \oint_{\partial \Gamma_{\varepsilon}} T_{i j} \theta_{\ell} \nu_{\ell} d s .
\end{aligned}
$$

We can now turn our attention to the integral on $\Gamma_{\varepsilon}$ in (47). To compute the derivative with respect to $p$, for fixed $\varepsilon$, we can employ again the general relation (13), simplified by the fact that $\boldsymbol{\theta} \cdot \mathbf{n}=0$ on $\Gamma_{\varepsilon}$

$$
\begin{aligned}
\left.\frac{d}{d p}\left(\int_{\Gamma_{\varepsilon}} T_{i j} u_{j} d S\right)\right|_{p=0}= & \int_{\Gamma_{\varepsilon}} T_{i j} \dot{u}_{j} d S+\int_{\Gamma_{\varepsilon}} \dot{T}_{i j} u_{j} d S+\oint_{\partial \Gamma_{\varepsilon}} T_{i j} u_{j} \theta_{\ell} \nu_{\ell} d s \\
= & \int_{\Gamma_{\varepsilon}} T_{i j} \dot{u}_{j} d S-a_{i} \int_{\Gamma_{\varepsilon}}\left(\Sigma_{i j k, \ell} n_{k}\right) u_{j} d S \\
& +u_{j}(\mathbf{y}) \oint_{\partial \Gamma_{\varepsilon}} T_{i j} \theta_{\ell} \nu_{\ell} d s+\oint_{\partial \Gamma_{\varepsilon}} T_{i j}\left[u_{j}-u_{j}(\mathbf{y})\right] \theta_{\ell} \nu_{\ell} d s \\
= & \int_{\Gamma_{\varepsilon}} T_{i j} \dot{u}_{j} d S+a_{\ell} \int_{\Gamma_{\varepsilon}} V_{i j \ell} u_{j} d S \\
& +u_{j}(\mathbf{y}) \oint_{\partial \Gamma_{\varepsilon}} T_{i j} \theta_{\ell} \nu_{\ell} d s+a_{\ell} \oint_{\partial \Gamma_{\varepsilon}} T_{i j}\left[u_{j}-u_{j}(\mathbf{y})\right] \nu_{\ell} d s+o(1),
\end{aligned}
$$

where $\dot{T}_{i j}=a_{\ell} V_{i j \ell}$.

It is worth noting that when $i \neq j$, and contrary to the potential case (cfr. (45)), the final line integral in the above expression may not be zero in the limit. In Appendix B it is shown that, when 
$\mathbf{y}$ is between two boundary elements with border of tangent $\mathbf{a}$, we obtain the following result $(i \neq j)$

$$
\lim _{\varepsilon \rightarrow 0} \oint_{\partial \Gamma_{\varepsilon}} T_{i j}\left[u_{j}-u_{j}(\mathbf{y})\right]\left(a_{\ell} \nu_{\ell}\right) d s=\frac{(1-2 \nu)}{12 \pi(1-\nu)}\left[\left(\frac{\partial \mathbf{u}}{\partial b^{1}} \times \mathbf{b}^{1}\right)_{i}-\left(\frac{\partial \mathbf{u}}{\partial b^{2}} \times \mathbf{b}^{2}\right)_{i}\right],
$$

where, on each side 1 and $2, \mathbf{b}^{\ell}=\mathbf{n}^{\ell} \times \mathbf{a}$ is a unit vector normal to the edge and tangent to the surface. It is clear that the result in (60) is zero when at $\mathbf{y}$ the boundary is smooth and the gradient $\nabla \mathbf{u}$ is continuous. Smoothness of the boundary is, in general, not sufficient since only $\partial \mathbf{u} / \partial a$ is continuous across the two elements.

When contributions from $s_{\varepsilon}$ and $\Gamma_{\varepsilon}$ are taken together, we observe that in (58) and (59) there are two line integrals that cancel each other. Therefore, we obtain the following final expression, if $\mathbf{u} \in C^{1, \alpha}(\mathbf{y})$

$$
\begin{aligned}
& \left.\frac{d I}{d p}\right|_{p=0}=\left.\lim _{\varepsilon \rightarrow 0} \frac{d}{d p}\left[\int_{\Gamma_{\varepsilon}\left(\mathbf{y}^{p}\right)+s_{\varepsilon}\left(\mathbf{y}^{p}\right)} T_{i j} u_{j} d S\right]\right|_{p=0} \\
& =\lim _{\varepsilon \rightarrow 0}\left[{ }^{*} u_{j}(\mathbf{y}) \int_{s_{\varepsilon}} T_{i j} d S+\int_{\Gamma_{\varepsilon}} T_{i j} \dot{u}_{j} d S+u_{j}(\mathbf{y}) \int_{s_{\varepsilon}} \dot{T}_{i j} d S+\int_{\Gamma_{\varepsilon}} \dot{T}_{i j} u_{j} d S\right] \\
& =\lim _{\varepsilon \rightarrow 0}\left[\left(\dot{u}_{j}(\mathbf{y})+\frac{\partial u_{j}}{\partial a}\right) \int_{s_{\varepsilon}} T_{i j} d S+\int_{\Gamma_{\varepsilon}} T_{i j} \dot{u}_{j} d S+u_{j}(\mathbf{y}) a_{\ell} \int_{s_{\varepsilon}} V_{i j \ell} d S+a_{\ell} \int_{\Gamma_{\varepsilon}} V_{i j \ell} u_{j} d S\right] \\
& =\dot{u}_{j}(\mathbf{y}) c_{i j}(\mathbf{y})+\frac{\partial u_{j}}{\partial a} c_{i j}(\mathbf{y})+f_{\partial \Omega} T_{i j} \dot{u}_{j} d S+\lim _{\varepsilon \rightarrow 0}\left[a_{\ell} \int_{\Gamma_{\varepsilon}} V_{i j \ell} u_{j} d S+u_{j}(\mathbf{y}) a_{\ell}\left(\frac{b_{i j \ell}}{\varepsilon}+d_{i j \ell}\right)\right] .
\end{aligned}
$$

If $\mathbf{u} \notin C^{1, \alpha}(\mathbf{y})$, the term in (60) must be added on the r.h.s.

\subsection{Some general remarks}

In the analysis presented in this paper the density functions $q, u, t_{j}$ and $u_{j}$ are just sufficiently regular functions that do not necessarily solve a boundary value problem as in the case, e.g., of approximate or numerical solutions. In particular, Green's or Betti's reciprocity identities have never been used. Moreover, the resulting formulae for the tangential derivatives of the integral $I(p)$ allow for a change of density $(\dot{q}, \dot{u}$, etc.) induced by the change of the singular point.

Among other things, these results support the approach presented in [8] to BEM error estimation through sensitivity analysis as shown in Section 8.

Quite remarkable is the fairly weak requirement posed on the smoothness of $u$ and $u_{j}$ at the singular point $\mathbf{y}$. The presented analysis only needs the density $u$ and its total derivative $\stackrel{*}{u}$ (eq. (39)) be $C^{0, \alpha}$ at the singular points. In other words, we do not require $u \in C^{1, \alpha}(\mathbf{y})$, but only $\dot{u}$ and its directional derivative $\partial u / \partial a$ to be Hölder continuous at y. As already stated, directional derivatives are continuous between two standard boundary elemements provided $\mathbf{a}$ is taken tangent to 
the interelement border. Therefore, the analysis is applicable when the point $\mathbf{y}$ is located on an edge and simply continuous elements are used.

\section{Numerical examples}

Some numerical computations are carried out to test the theoretical findings presented in this paper. In particular, we consider formula (61) for the tangential derivative of a double-layer elastic potential, which is probably the most severe test.

Formula (61) covers the general case. However, if we make some simplifying assumptions, the same computation can also be performed by alternative classical means that can be used for comparison.

In particular, if we consider a piece-wise flat surface $\Gamma$, the kernel function $T_{i j}$ becomes

$$
T_{i j}(\mathbf{y}, \mathbf{x})=-\frac{(1-2 \nu)}{8 \pi(1-\nu)} \frac{\left(n_{i} r_{, j}-n_{j} r_{, i}\right)}{r^{2}}=\frac{(1-2 \nu)}{8 \pi(1-\nu)} D_{i j}\left(\frac{1}{r}\right),
$$

and hence, according to (5), the following Cauchy principal value integral can be converted into a line integral

$$
f_{\Gamma} T_{i j} d S=\lim _{\varepsilon \rightarrow 0} \int_{\Gamma_{\varepsilon}\left(\mathbf{y}^{p}\right)} T_{i j}\left(\mathbf{y}^{p}, \mathbf{x}\right) d S_{x}=\frac{(1-2 \nu)}{8 \pi(1-\nu)} \lim _{\varepsilon \rightarrow 0} \oint_{\partial \Gamma_{\varepsilon}} e_{i j k} \tau_{k}(\mathbf{x}) \frac{d s}{r},
$$

where $\partial \Gamma_{\varepsilon}=\partial \Gamma \cup \partial e_{\varepsilon}$ (Figure 1). If the singular point lies on the edge between two panels $\Gamma_{1}$ and $\Gamma_{2}$ we obtain that

$$
I(\mathbf{y})=f_{\Gamma} T_{i j} d S=\frac{1-2 \nu}{8 \pi(1-\nu)} \oint_{\partial \Gamma} e_{i j k} \tau_{k}(\mathbf{x}) \frac{d s}{r}+2 e_{i j k}\left(\mathbf{b}^{1}-\mathbf{b}^{2}\right) \cdot \mathbf{e}_{k},
$$

where, on each panel $\Gamma_{\ell}, \mathbf{b}^{\ell}=\mathbf{n}^{\ell} \times \mathbf{a}$ like in (60). If the source point $\mathbf{y}$ lies within one panel, the last term vanishes.

In either case, the line integrals on the r.h.s. involve only regular functions since $\mathbf{y} \notin \partial \Gamma$. Thus, differentiation with respect to $\mathbf{y}$ can be expressed by differentiation under the integral sign. If, as usual in this paper, $y^{p}=\mathbf{y}(p)=\mathbf{y}+\mathbf{a} p+\mathbf{o}(p)$, we have that

$$
\left.\frac{d I}{d p}\right|_{p=0}=\frac{1-2 \nu}{8 \pi(1-\nu)} \oint_{\partial \Gamma} e_{i j k} \tau_{k} a_{\ell} \frac{\left(x_{\ell}-y_{\ell}\right)}{r^{3}} d s .
$$

The regular integral on the r.h.s. can be evaluated by standard quadrature rules.

The last result is precisely what we need to evaluate the derivative in (61) if we assume a constant density $u_{j}=1$ (and hence $\mathbf{u} \in C^{1, \alpha}(\mathbf{y})$ ), with $\dot{u}_{j}=0$. Therefore, we can compare these results to check the general formula (61) which employs the hypersingular integration algorithm described in [9] and [10]. In particular, we can test the exactness of our results regarding the differentiation along the edge. 


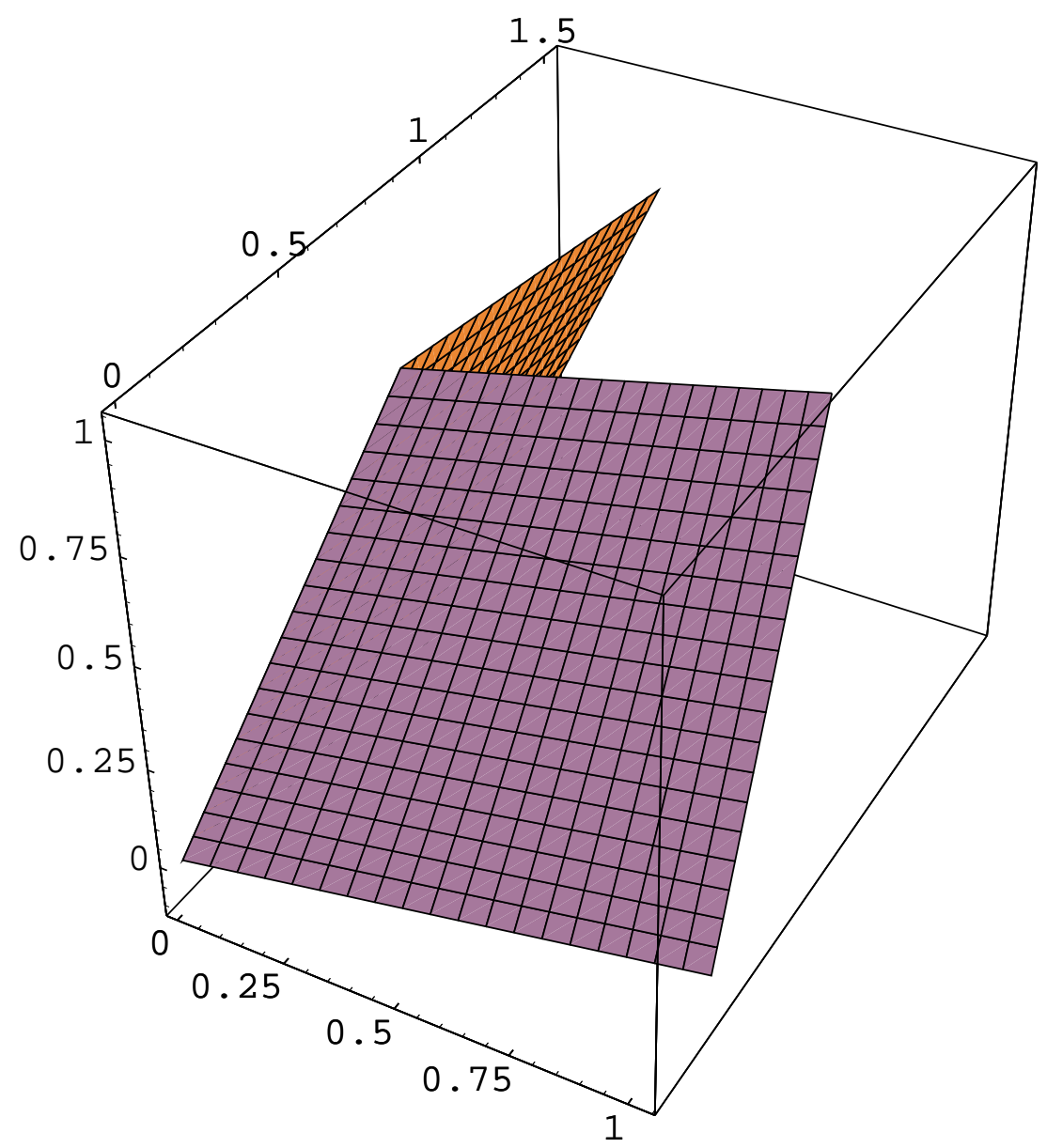

Figure 2: Flat distorted elements employed in numerical examples.

Two flat panels of distorted shape and general orientation were considered (Figure 2). The coordinates of the four nodes of each element are as follows: $(1.03,0.65,1.03),(0.3,0.4,1),(0,0,0)$, $(1,0.3,-0.1)$ for one element, and $(0.3,0.4,1),(0.24,1.52,0.8),(0,1,0),(0,0,0)$, for the other element. The singular point $\mathbf{y}=(0.189,0.252,0.63)$ was on the common edge of direction $\mathbf{a}=$ $(0.268328,0.357771,0.894427)$. A Poisson ratio $\nu=0.3$ was assumed.

Both methods, i.e., equations (61) and (62), provided the same results up to the seventh significant digit, thus confirming the correctness of the theoretical results presented even when the singular point is located on an edge. The numerical results are summarized in Table 1 for all relevant indices of the kernel function $T_{i j}$ (kernels with equal indices are not singular on flat elements).

For completeness, the same comparison was carried out also for the case of the singular point $y$ located inside one flat element. Again, for more generality, the element had a distorted shape. The coordinates of the four nodes are as in the first element of the former example. The singular point 
Table 1: Derivatives of strongly singular integrals: pole along the edge between two elements.

\begin{tabular}{||c|c||}
\hline indices $(i, j)$ & derivative \\
\hline$(1,2)$ & -0.00277012 \\
$(2,3)$ & -0.0719483 \\
$(3,1)$ & 0.0590433 \\
\hline
\end{tabular}

Table 2: Directional derivatives of strongly singular integrals: pole within one element.

\begin{tabular}{||c|c||}
\hline indices $(i, j)$ & derivative \\
\hline$(1,2)$ & 0.113809 \\
$(2,3)$ & -0.0711122 \\
$(3,1)$ & 0.0107791 \\
\hline
\end{tabular}

$y$ was set to have intrinsic c oordinates $(0.3,-0.2)$, and the derivative was taken along the direction $\mathbf{a}=(0.645733,0.407501,0.645733)$. The results obtained with both methods, i.e., equations (61) and (62), agreed within six significant digits and are reported in Table 2.

\section{Sensitivity of approximate boundary element solutions}

In the standard boundary element method (BEM), approximate solutions are obtained by collocation at a suitable number of boundary points. Details can be found in any BEM book.

Let, as in [8], the whole set of collocation points $\left\{\mathbf{y}^{(k)}\right\}$ be dependent upon a single scalar parameter $p$. Therefore, given the value of $p$ we know the location of each collocation point $\mathbf{y}^{(k)}(p)$ on $\partial \Omega$. The value $p=0$ can be associated to the standard nodal collocation. Clearly, the approximate BEM solution $u_{j}\left(\left\{\mathbf{y}^{(k)}(p)\right\}, \mathbf{x}\right)=u_{j}(p, \mathbf{x})$ and $t_{j}\left(\left\{\mathbf{y}^{(k)}(p)\right\}, \mathbf{x}\right)=t_{j}(p, \mathbf{x})$ do depend on $p$ through the full set $\left\{\mathbf{y}^{(k)}(p)\right\}$ of $p$-dependent collocation points.

After the system of equations has been solved, the approximate solution is known all over the boundary $\partial \Omega$ and we have the following identities

$$
\lim _{\varepsilon \rightarrow 0} \int_{\partial \Omega_{\varepsilon}\left(\mathbf{y}^{(k)}(p)\right)}\left[T_{i j}\left(\mathbf{y}^{(k)}(p), \mathbf{x}\right) u_{j}(p, \mathbf{x})-U_{i j}\left(\mathbf{y}^{(k)}(p), \mathbf{x}\right) t_{j}(p, \mathbf{x})\right] d S=R_{k}(p) \equiv 0,
$$

where $\mathbf{y}^{(k)}(p)$ is any collocation point. Some comments are in order here. First, we must observe that $R_{k}$ is not the residual of the boundary integral equation. The residual would be obtained with a fixed approximate solution and a changing singular point, whereas both depend on $p$ in (63). In fact, $R_{k}(p)$ is, by construction, identically zero for any $p$. Changing $p$ means selecting a new set of collocation 
points and obtaining, by collocation and through the solution of a new system of equations, a different numerical solution which has again zero residual at each new point $\mathbf{y}^{(k)}(p)$.

Obviously, since $R_{k}(p) \equiv 0$, also its derivative is identically zero

$$
\frac{d R_{k}}{d p} \equiv 0
$$

The results obtained in this paper allow for a more explicit expression of the above derivative. It is just a matter of collecting the results in (30) and (61)

$$
\begin{aligned}
\lim _{\varepsilon \rightarrow 0} \frac{d}{d p} \int_{\partial \Omega_{\varepsilon}\left(\mathbf{y}^{(k)}(p)\right)}\left[T_{i j}\left(\mathbf{y}^{(k)}(p), \mathbf{x}\right) u_{j}(p, \mathbf{x})-U_{i j}\left(\mathbf{y}^{(k)}(p), \mathbf{x}\right) t_{j}(p, \mathbf{x})\right] d S \\
=c_{i j}(\mathbf{y}) \dot{u}_{j}(\mathbf{y})+\int_{\partial \Omega} T_{i j} \dot{u}_{j} d S-\int_{\partial \Omega} U_{i j} \dot{t}_{j} d S \\
\quad+c_{i j}(\mathbf{y}) \frac{\partial u_{j}}{\partial a}+a_{\ell} d_{i j \ell} u_{j}(\mathbf{y})+\lim _{\varepsilon \rightarrow 0}\left[\int_{\Gamma_{\varepsilon}} a_{\ell}\left[V_{i j \ell} u_{j}-W_{i j \ell} t_{j}\right] d S+a_{\ell} \frac{b_{i j \ell}}{\varepsilon} u_{j}(\mathbf{y})\right] \equiv 0,
\end{aligned}
$$

where $\mathbf{y}=\mathbf{y}^{(k)}(p)$. If $u_{j} \notin C^{1, \alpha}\left(\mathbf{y}^{(k)}\right)$, the additional term (60) must be added on the r.h.s.

Expression (64) supports the approach presented in [8] where the sensitivity of two-dimensional approximate boundary element solutions with respect to the positions of collocation points was obtained. Each row in the system of equations (14) in [8] is identical to eq. (64), provided the BEM solution is used for the density functions $u_{j}$ and $t_{j}$. (The notation is different: dotted quantities in this paper correspond to starred quantities in the other paper).

If we rewrite the identity (64) in this fashion

$$
\begin{aligned}
& c_{i j}(\mathbf{y}) \dot{u}_{j}(\mathbf{y})+f_{\partial \Omega} T_{i j} \dot{u}_{j} d S-\int_{\partial \Omega} U_{i j} \dot{t}_{j} d S \\
& \quad=-\left\{c_{i j}(\mathbf{y}) \frac{\partial u_{j}}{\partial a}+a_{\ell} d_{i j \ell} u_{j}(\mathbf{y})+\lim _{\varepsilon \rightarrow 0}\left[\int_{\Gamma_{\varepsilon}} a_{\ell}\left[V_{i j \ell} u_{j}-W_{i j \ell} t_{j}\right] d S+a_{\ell} \frac{b_{i j \ell}}{\varepsilon} u_{j}(\mathbf{y})\right]\right\} .
\end{aligned}
$$

we have on the l.h.s. a standard boundary integral equation for the "sensitivities" $\dot{u}$ and $\dot{t}$, and on the r.h.s. the residual (with minus sign) of the hypersingular boundary integral equation (HBIE) for the tangential derivatives in the a-direction.

The functions $\dot{u}$ and $\dot{t}$ show that the approximate solution is, in fact, sensitive to the selection of collocation points whenever the same approximate solution fails to satisfy the HBIE for tangential derivatives. The exact solution would have all dotted functions identically equal to zero since the r.h.s. would be identically zero as well.

In [8] it was heuristically suggested to employ suitable norms of these sensitivities to steer a self adaptive mesh refinement process. 


\section{Acknowledgments}

This work was carried out while M. Bonnet was visiting the University of Pisa (Italy) sponsored by the French Academy of Sciences. Support to M. Guiggiani was provided by MURST.

\section{References}

[1] Bonnet, M. Equations intégrales et éléments de frontière.. CNRS Editions / Eyrolles, Paris, France (1995).

[2] Bonnet, M. Regularized BIE formulations for first- and second-order shape sensitivity of elastic fields. Computers and Structures, 56, 799-811 (1995). (Invited paper, special issue, S. Saigal, guest editor).

[3] Bonnet, M. Differentiability of strongly singular and hypersingular boundary integral formulations with respect to boundary perturbations. Comp. Mech., 19, 240-246 (1997).

[4] Bui, H. D. Some remarks about the formulation of three-dimensional thermoelastoplastic problems by integral equations. Int. J. Solids Struct., 14, 935-939 (1978).

[5] Guiggiani, M. Error indicators for adaptive mesh refinement in the Boundary Element Method-A new approach. Int. J. Num. Meth. in Eng., 29, 1247-1269 (1990).

[6] Guiggiani, M. Hypersingular formulation for boundary stress evaluation. Engng. Anal. with Bound. Elem., 14, 169-179 (1994).

[7] Guiggiani, M. Hypersingular boundary integral equations have an additional free term. Comp. Mech., 16, 245-248 (1995).

[8] Guiggiani, M. Sensitivity analysis for boundary element error estimation and mesh refinement. Int. J. Num. Meth. in Eng. (1996).

[9] Guiggiani, M., Krishnasamy, G., Rizzo, F.J., Rudolphi, T.J. Hypersingular boundary integral equations: a new approach to their numerical treatment. In L. Morino, R. Piva (eds.), Boundary Integral Methods, pp. 211-220. Springer-Verlag (1991).

[10] Guiggiani, M., Krishnasamy, G., Rudolphi, T. J., Rizzo, F. J. A general algorithm for the numerical solution of hypersingular boundary integral equations. ASME J. Appl. Mech., 59, 604-614 (1992). 
[11] KellogG, O. D. Fundations of potential theory. Springer-Verlag, Berlin, Heidelberg (1967).

[12] Kupradze, V. D. (ed.). Three-dimensional problems of the mathematical theory of elasticity and thermoelasticity. North Holland (1979).

[13] Petryk, H., Mróz, Z. Time derivatives of integrals and functionals defined on varying volume and surface domains. Arch. Mech., 38, 694-724 (1986).

[14] Zhang, Q., Mukherjee, S. Second-order design sensitivity analysis for linear elastic problems by the derivative boundary element method. Comp. Meth. in Appl. Mech. Engng., 86, 321-335 (1991).

\section{Appendix A: Existence of the limits}

The integrals in (24) whose value might be affected by the shape of the exclusion neighbourhood are those with more singular kernels. For simplicity, the analysis is presented for smooth boundary at $\mathbf{y}$. However, the same path can be followed when $\mathbf{y}$ lies on an edge and $q$ is only piecewise $C^{0, \alpha}$.

Basically, using (2) and then integrating by parts using eq. (6) we have that

$$
\begin{aligned}
\int_{\Gamma_{\varepsilon}} \frac{\partial G}{\partial y_{m}} d S+\oint_{\partial \Gamma_{\varepsilon}} G \nu_{m} d s & =\int_{\Gamma_{\varepsilon}}-\left(D_{m} G-n_{m} \frac{\partial G}{\partial n}\right) d S+\oint_{\partial \Gamma_{\varepsilon}} G \nu_{m} d s \\
& =\int_{\Gamma_{\varepsilon}} n_{m}\left(2 \kappa G+\frac{\partial G}{\partial n}\right) d S-\oint_{\partial \Gamma_{\varepsilon}} G \nu_{m} d s+\oint_{\partial \Gamma_{\varepsilon}} G \nu_{m} d s
\end{aligned}
$$

where $\kappa$ is the mean curvature. In the last equation, the original singular integrals have been converted into a weakly singular integral on $\Gamma_{\varepsilon}$. Thus the limit investigated exists and its value does not depend on the shape of $v_{\varepsilon}(\mathbf{y})$.

Now, let us consider eq. (46). We recall that the third line in (46) follows from the first or second line only when a circular shape of uniform radius $\varepsilon$ is taken for the vanishing neighbourhood $v_{\varepsilon}$. We have therefore to show that this choice for $v_{\varepsilon}$ is not relevant.

Integrating by parts as in (5) we have that

$$
\begin{aligned}
-a_{i} \int_{\Gamma_{\varepsilon}}\left(G, i j n_{j}\right) u d S & =a_{i} \int_{\Gamma_{\varepsilon}}\left(D_{i j} G,,_{j}\right) u d S \\
& =a_{i} e_{i j p} \oint_{\partial \Gamma_{\varepsilon}} \tau_{p} G,{ }_{j} u d s+a_{i} \int_{\Gamma_{\varepsilon}} G,,_{j}\left(D_{j i} u\right) d S \\
& =\oint_{\partial \Gamma_{\varepsilon}}\left[\left(a_{i} n_{i}\right) \nu_{j}-\left(a_{i} \nu_{i}\right) n_{j}\right] G,,_{j} u d s+a_{i} \int_{\Gamma_{\varepsilon}} G,,_{j}\left(D_{j i} u\right) d S .
\end{aligned}
$$


Still by integration by parts we already knew that

$$
-\int_{s_{\varepsilon}} G,{ }_{i j} n_{j} d S=\oint_{\partial \Gamma_{\varepsilon}} G,,_{j}\left(\nu_{i} n_{j}-\nu_{j} n_{i}\right) d s .
$$

Therefore, an alternative expression for (46) is

$$
\begin{aligned}
\left.\lim _{\varepsilon \rightarrow 0} \frac{d}{d p}\left[\int_{\Gamma_{\varepsilon}\left(\mathbf{y}^{p}\right)+s_{\varepsilon}\left(\mathbf{y}^{p}\right)} T u d S\right]\right|_{p=0} \\
=\lim _{\varepsilon \rightarrow 0}\left[\stackrel{*}{u}(\mathbf{y}) \int_{s_{\varepsilon}} G,{ }_{j} n_{j} d S+\int_{\Gamma_{\varepsilon}}\left(G,{ }_{j} n_{j}\right) \dot{u} d S+u(\mathbf{y}) a_{i} \oint_{\partial \Gamma_{\varepsilon}} G,,_{j}\left(\nu_{i} n_{j}-\nu_{j} n_{i}\right) d s\right. \\
\left.+\oint_{\partial \Gamma_{\varepsilon}}\left[\left(a_{i} n_{i}\right) \nu_{j}-\left(a_{i} \nu_{i}\right) n_{j}\right] G,,_{j} u d s+a_{i} \int_{\Gamma_{\varepsilon}} G,,_{j}\left(D_{j i} u\right) d S+a_{i} \oint_{\partial \Gamma_{\varepsilon}} G,{ }_{j} n_{j}[u-u(\mathbf{y})] \nu_{i} d s\right] \\
=\lim _{\varepsilon \rightarrow 0}\left[\stackrel{*}{u}(\mathbf{y}) \int_{s_{\varepsilon}} G,{ }_{j} n_{j} d S+\int_{\Gamma_{\varepsilon}}\left(G,{ }_{j} n_{j}\right) \dot{u} d S+a_{i} \int_{\Gamma_{\varepsilon}} G,{ }_{j}\left(D_{j i} u\right) d S\right. \\
\left.\quad+\oint_{\partial \Gamma_{\varepsilon}} G,{ }_{j} \nu_{j}\left(a_{i} n_{i}\right)[u-u(\mathbf{y})] d s\right],
\end{aligned}
$$

where the final line integral clearly tends to zero if $\boldsymbol{\theta} \cdot \mathbf{n}=0$ and $\mathbf{u} \in C^{0, \alpha}(\mathbf{y})$.

Further manipulations are possible since

$$
\int_{s_{\varepsilon}} G,{ }_{j} n_{j} d S=-\int_{\Gamma_{\varepsilon}} G,{ }_{j} n_{j} d S
$$

and

$$
a_{i} \int_{\Gamma_{\varepsilon}} G,,_{j}\left(D_{j i} u\right) d S=\int_{\Gamma_{\varepsilon}}\left[\left(a_{i} u,,_{i}\right) G,{ }_{j} n_{j}-\left(a_{i} n_{i}\right) G,{ }_{j} u,{ }_{j}\right] d S,
$$

where $a_{i} n_{i}=O\left(\varepsilon^{\alpha}\right)$. Moreover, we can set

$$
\begin{aligned}
a_{i} u,_{i} & =a_{i}\left[u,_{i}-u,_{i}(\mathbf{y})\right]+a_{i} u,_{i}(\mathbf{y}) \\
& =a_{i}\left[u,_{i}-u,_{i}(\mathbf{y})\right]+\dot{u}(\mathbf{y})+a_{i} u,_{i}(\mathbf{y})-\dot{u}(\mathbf{y}) \\
& =a_{i}\left[u,_{i}-u,_{i}(\mathbf{y})\right]+\stackrel{*}{u}(\mathbf{y})-\dot{u}(\mathbf{y})
\end{aligned}
$$

Summing up, the following regularized expression for (A3) or (46) is obtained

$$
\begin{aligned}
\left.\lim _{\varepsilon \rightarrow 0} \frac{d}{d p}\left\{\int_{\Gamma_{\varepsilon}+s_{\varepsilon}} T u d S\right\}\right|_{p=0} & =\lim _{\varepsilon \rightarrow 0} \int_{\Gamma_{\varepsilon}}\left\{\left([\dot{u}-\dot{u}(\mathbf{y})]+a_{i}\left[u,,_{i}-u,_{i}(\mathbf{y})\right]\right) G,_{j} n_{j}-\left(a_{i} n_{i}\right) G,,_{j} u,,_{j}\right\} d S \\
& =\int_{\partial \Omega}\left\{\left([\dot{u}-\dot{u}(\mathbf{y})]+a_{i}\left[u,_{i}-u,,_{i}(\mathbf{y})\right]\right) G,,_{j} n_{j}-\left(a_{i} n_{i}\right) G,,_{j} u,,_{j}\right\} d S(\mathrm{~A} 4)
\end{aligned}
$$

Since all integrals are weakly singular the limit exists and it does not depend on the selected shape of $v_{\varepsilon}$.

Similar proofs can be provided for the elastic case. 


\section{Appendix B: Proof of eq. (60)}

Let the singular point $\mathbf{y}$ be on an edge of tangent $\mathbf{a}$. On each side we define a unit vector $\mathbf{b}^{\ell}, \ell=1,2$

$$
\mathbf{b}^{\ell}=\mathbf{n}^{\ell} \times \mathbf{a}
$$

normal to the edge and tangent to the surface. Clearly, $\left(\mathbf{a}, \mathbf{b}^{\ell}, \mathbf{n}^{\ell}\right), \ell=1,2$, are two Cartesian orthonormal bases.

Let $(\mathbf{a}, \mathbf{b}, \mathbf{n})=\left(\mathbf{a}, \mathbf{b}^{1}, \mathbf{n}^{1}\right)$ be the basis with $\mathbf{b}$ pointing out of the edge. We have on the half part $\partial \hat{\Gamma}_{\varepsilon}$ of $\partial \Gamma_{\varepsilon}$ lying on the corresponding element

$$
\begin{aligned}
r & =\varepsilon \\
r, 1 & =\cos \theta+O(\varepsilon) \\
r, 2 & =\sin \theta+O(\varepsilon) \\
r_{3} & =O(\varepsilon) \\
n_{\ell}(\mathbf{y}) & =\delta_{\ell 3} \\
d s & =\varepsilon(1+O(\varepsilon)) d \theta \\
\nu_{\ell} & =-r, \ell+O(\varepsilon) \\
\mathbf{a} & =(1,0,0) \\
\nu_{\ell} a_{\ell} & =-\cos \theta+O(\varepsilon),
\end{aligned}
$$

where polar coordinated centered at $\mathbf{y}$ have been introduced.

Assume that on each side of the edge (i.e., for $0 \leq \theta \leq \pi$ here), $\mathbf{u}$ has a $C^{0, \alpha}$ gradient. Then

$$
u_{j}-u_{j}(\mathbf{y})=\varepsilon u_{j, m}(\mathbf{y}) r, m(\mathbf{y})+O\left(\varepsilon^{1+\alpha}\right) .
$$

Then, one has

$$
\oint_{\partial \hat{\Gamma}_{\varepsilon}} T_{i j}\left[u_{j}-u_{j}(\mathbf{y})\right]\left(a_{\ell} \nu_{\ell}\right) d s=\int_{0}^{\pi} \frac{-(1-2 \nu)}{8 \pi(1-\nu) \varepsilon^{2}}\left[r,{ }_{j} n_{i}-r,{ }_{i} n_{j}\right] \varepsilon u_{j, m}(\mathbf{y}) r,,_{m}(-\cos \theta) \varepsilon d \theta+o(1)
$$

where $n_{\ell}=n_{\ell}(\mathbf{y})$ and $\nu$ is the Poisson ratio. Now using

$$
\int_{0}^{\pi}\left(\cos ^{3} \theta, \cos ^{2} \theta \sin \theta, \cos \theta \sin ^{2} \theta\right) d \theta=(0,2 / 3,0)
$$

we have

$$
\int_{0}^{\pi} r_{, i} r_{m} \cos \theta d \theta=(2 / 3)\left(\delta_{i 1} \delta_{m 2}+\delta_{i 2} \delta_{m 1}\right)
$$


so that

$$
\begin{aligned}
\lim _{\varepsilon \rightarrow 0} \oint_{\partial \hat{\Gamma}_{\varepsilon}} T_{i j}\left[u_{j}-u_{j}(\mathbf{y})\right]\left(a_{\ell} \nu_{\ell}\right) d s & =A\left[\left(\delta_{j 1} \delta_{m 2}+\delta_{j 2} \delta_{m 1}\right) \delta_{i 3}-\left(\delta_{i 1} \delta_{m 2}+\delta_{i 2} \delta_{m 1}\right) \delta_{j 3}\right] u_{j, m}(\mathbf{y}) \\
& =A\left[\left(\delta_{i 3} \delta_{j 1}-\delta_{i 1} \delta_{j 3}\right) \delta_{m 2}+\left(\delta_{i 3} \delta_{j 2}-\delta_{i 2} \delta_{j 3}\right) \delta_{m 1}\right] u_{j, m}(\mathbf{y}) \\
& =A\left[e_{k i j} e_{k 31} u_{j, 2}(\mathbf{y})+e_{k i j} e_{k 32} u_{j, 1}(\mathbf{y})\right] \\
& =A\left[(\mathbf{u}, 2 \times \mathbf{b})_{i}-(\mathbf{u}, 1 \times \mathbf{a})_{i}\right],
\end{aligned}
$$

where $A=(1-2 \nu) /(12 \pi(1-\nu))$ and the derivatives of $\mathbf{u}$ are intended in the local frame $(\mathbf{a}, \mathbf{b}, \mathbf{n})$.

Since $\mathbf{u}, 1$ is continuous across the edge, the contribution for the two half-circles add up to zero. On the other hand, the contributions of terms involving $\left(\mathbf{u},_{2} \times \mathbf{b}\right)_{i}$ will give rise to nonzero free terms in eq. (60) which depend on the two derivatives of $\mathbf{u}$ along the directions $\mathbf{b}^{\ell}$. 